\title{
SQP-based Power Allocation Strategy for Target Tracking in MIMO Radar Network with Widely Separated Antennas
}

\author{
Mohammad Akhondi Darzikolaei \\ Department of Electrical and Computer Engineering, Babol Noshirvani University of Technology,Babol, Iran \\ M.akhondi@stu.nit.ac.ir \\ Mohammad Reza Karami Mollaei * \\ Department of Electrical and Computer Engineering, Babol Noshirvani University of Technology,Babol, Iran \\ mkarami@nit.ac.ir \\ Maryam Najimi \\ Department of Electrical and Computer Engineering, University of Science and Technology of Mazandaran,Behshahr, Iran \\ m.najimi@mazust.ac.ir
}

Received: 22/Aug/2021_ Revised: 06/Nov/2021_Accepted: 07/Dec/2021

\begin{abstract}
MIMO radar with widely separated antennas enhances detection and estimation resolution by utilizing the diversity of the propagation path. Each antenna of this type of radar can steer its beam independently towards any direction as an independent transmitter. However, the joint processing of signals for transmission and reception differs this radar from the multistatic radar. There are many resource optimization problems which improve the performance of MIMO radar. But power allocation is one of the most interesting resource optimization problems. The power allocation finds an optimum strategy to assign power to transmit antennas with the aim of minimizing the target tracking errors under specified transmit power constraints. In this study, the performance of power allocation for target tracking in MIMO radar with widely separated antennas is investigated. Therefore, a MIMO radar with distributed antennas is configured and a target motion model using the constant velocity (CV) method is modeled. Then Joint Cramer Rao bound (CRB) for target parameters (joint target position and velocity) estimation error is calculated. This is utilized as a power allocation problem objective function. Since the proposed power allocation problem is nonconvex. Therefore, a SQP-based power allocation algorithm is proposed to solve it. In simulation results, the performance of the proposed algorithm in various conditions such as a different number of antennas and antenna geometry configurations is examined. Results affirm the accuracy of the proposed algorithm.
\end{abstract}

Keywords: MIMO radar; Power allocation; SQP; Target tracking.

\section{1- Introduction}

The RADAR is a short form of Radio Detection And Ranging. Radar utilizes electromagnetic waves to detect, locate and measure the speed of reflected objects. It transmits the electromagnetic waves into space and receives the echo signals [1-2]. In recent years, radar network systems such as multi-static radars and multi-input multioutput (MIMO) radars have become an attractive and improtant problem [3]. Spatial diversity [4], waveform diversity [5], and multiplexing gain [6] over common monostatic radar [7] are some positive characteristics of networked radar systems. This structure of radars helps to raise tracking accuracy in multiple target tracking scenarios with radar, sonar, and video sensors [8]. A MIMO radar is a kind of radar structure that uses a combination of antennas as transmitter and receiver and each of them emits its own waveform apart from others [9]. Widely separated antennas and collocated antennas are two famous categories for MIMO radar. In collocated MIMO radar, the antennas are close to each other. The antennas in MIMO radar with widely separated antennas are far from each other. In other words, the transmit and receive antennas are located in a wide area. Therefore, the target is seen from different angles by antennas. In this type of radars, each receiver should receive all signals from all transmitters and then emit them to the central processor. This means that each receiver does not process or make a decision individually and sends its signals to the central processor to process all signals. This feature is the main difference between multi-static and MIMO radar with widely separated antennas. Power allocation is usually performed in radar networks to find the

* Corresponding Author 
best strategy to assign power among various transmit antennas, aiming at minimizing the estimation error under specified transmit power constraints or its converse [10]. Power allocation is an important section of military operations in a hostile environment to obtain a low probability of interception [11]. Power allocation in radar networks is studied in last researches. For example, in [12] power allocation in widely separated multi-input multioutput (MIMO) radar for range-only target tracking is evaluated. This is performed by maximizing the Bayesian Fisher information matrix (B-FIM). B-FIM is derived for the predetermined signal model and then the problem is modeled as one cooperative game. [13] Shows the commercial application of power allocation in radar networks. In fact, it puts a cognitive radar network in an urban environment and this network tracks cars and vehicles. The power allocation problem for radar networks in a cooperative game-theoretic structure is considered in [14] to enhance the low probability of intercept (LPI) performance. In addition, by considering transmit power constraint and minimum signal to noise and interference ratio (SINR) for each radar, a cooperative Nash Bargaining power allocation game based on LPI is expressed. The radar network in [15] consists of unmodulated continuous wave (UCW) radars. This network utilizes a power allocation algorithm for Doppler-only target tracking. This algorithm minimizes the mean square error of target state estimation with a power budget constraint. [16] Investigates power allocation for radar networks to increase the performance of low probability of intercept. Two power allocation strategies are stated in this reference. One is for optimizing transmit power allocation with predetermined mutual information (MI) threshold and another is for finding optimal power allocation with minimum mean square error (MMSE) threshold. In [17], power allocation and target assignment in radar networks is mentioned to improve LPI performance. The target geometrical method is used to fuse information for measuring target localization by different radars. Authors in [18] propose joint antenna selection and power allocation for localization in distributed MIMO radar networks. The sensor management is performed by solving a constrained problem which is expressed to minimize the error in estimating target position, while it is constrained by transmitter number and power budget. [19] Talks about sensor selection in radar networks and for target tracking, the number of radars are selected. This problem is just for one target tracking. In this reference, sensor selection is performed by information theory. Joint transmitter and receiver selection in target tracking in distributed MIMO radar is described in [20]. Due to resource restriction in radars, it is necessary to select some radars in the MIMO radar network at each time and also keep the system performance in the best condition. So, lower bound PCRLB is $\mathrm{u}$ as an optimization criterion for an optimization problem in this literature. In [21], a joint power allocation and sensor selection algorithm for multi-target tracking in an LPI radar network with $\mathrm{N}$ monostatic radars is introduced. This algorithm can minimize the total transmitted power of a radar network based on predefined mutual information threshold between reflected signal and target impulse computed predictively with the estimation of the target state is needed for estimation of target parameters. [22] Describes the joint beam selection and power allocation strategies for multi-target tracking in collocated MIMO radar. Each radar works based on a multi-beam working mode, in which multiple simultaneous transmit beams are synthesized. This strategy applies an optimization technique to control the limited beam and power resource of each radar to obtain accurate target state estimation. Therefore, Bayesian Cramer Rao Lower Bound is extracted, normalized, and utilizes as the optimization criterion. To increase the system performance and resource utilization of widely separated MIMO radar, a joint resource allocation for velocity estimation problem in multi-target tracking is proposed in [23]. The authors chose one target as a key target and then examined their strategy by this target. They considered a Mean Square Estimation of velocity estimation of the key target as a minimization problem criterion. With limited resources and requirements for velocity estimation for targets, a joint optimization model with the selection of numbers of receivers and transmitters and allocation of transmit power and signal time is introduced. The Authors in [24] claims that since transmitters in MIMO radars with widely separated antennas emit waveforms with different powers and bandwidths, therefore these two parameters are limited. In this reference, they offer power allocation, bandwidth allocation, and joint power and bandwidth allocation problems. They compute Cramer Rao for target localization accuracy and utilize it as optimization criteria. In [25], a solution for joint beam and power scheduling in the netted Collocated MIMO radar systems for distributed multi-target tracking is suggested. An adaptive sensor scheduling integrated with power and bandwidth allocation is presented for centralized multiple target tracking in the netted collocated MIMO radar in [26]. 
By investigating the above references, we understand that power allocation for target tracking in MIMO radar with widely separated antennas is very essential and the performance of this problem should be improved. With our reviews, there are some challenges in the power allocation problem for target tracking in MIMO radar with widely separated antennas which are not investigated in other papers and we consider them in this paper.

1. In this paper, for the calculation of target tracking errors, joint target velocity and position estimation is used to improve tracking performance. Although someone works on this issue for MIMO radar, this is not used in power allocation problems in MIMO radar with widely separated antennas. For example, in [25] and [7], joint estimation is considered but certain power is determined for the transmit power and power allocation strategy is not performed. And also, in [25], it does not exactly specify its MIMO radar structure (distributed or collocated). In other researches about power allocation for target tracking in MIMO radar with widely separated antennas, joint estimation is not worked. For instance, in [12], range-only estimation for target tracking in MIMO radar with widely separated antennas is performed and it is not considered velocity. Whereas, in [23], velocity estimation is utilized to compute target tracking for power allocation problem in distributed MIMO radar. These are just some papers which we investigated and we concluded that joint estimation for target tracking in power allocation problem for MIMO radar with widely separated antennas is not used yet. Although it may be performed for collocated MIMO radar. Thus, joint target velocity and position estimation is the first novel idea for our power allocation strategy in MIMO radar with widely separated antennas.

2. Using random mathematical statistics for target RCS is another unique characteristic of this paper. Because in other researches, or a deterministic model for target RCS is considered [7, 24, 18, 22, 23], or if they supposed a random target RCS, they neglected it in their next calculations for simplicity [20, 21]. Therefore, none of the previous researches did contribute a power allocation strategy for target tracking in MIMO radar with widely separated antennas by considering random complex Gaussian target RCS. They usually neglected it or put a deterministic number instead of variance of Gaussian distribution. But in this paper, in all calculations, random complex Gaussian with random variance in different transmit-receive paths is considered. Note that assuming random RCS is necessary for MIMO radar with widely separated antennas. Because each antenna sees the target in a specified angle and target reflections in different transmit-receive paths are different with respect to each other.

The main contributions of this paper are as follows:

1. The system model for MIMO radar with widely separated antennas is introduced. Then $\mathrm{CV}$ model is considered for target motion. Considering a complex Gaussian random model for target RCS and using this feature in the next calculations, Cramer Rao bound for target parameters estimation error, is one of the prominent aspects of this paper.

2. Maximum likelihood (ML) estimation for unknown target parameters which were target position and velocity is calculated and then joint CRB for target position and velocity estimation is computed. Output of joint CRB is considered as an objective function for power allocation problem.

3. A power allocation strategy is formed. In fact, joint CRB for target position and velocity estimation function (target tracking errors) subject to some constraints such as limitation in total transmit power and transmit power of each transmit antenna is the power allocation problem of this paper. Our goal is to minimize tracking errors by using the mentioned constraints.

4. For solving the previous section problem, since it is nonconvex problem, $\mathrm{SQP}^{1}$ based power allocation algorithm is proposed. This algorithm is formed based on the SQP algorithm and it can allot optimal power to each transmit antenna to satisfy the constraints in the problem. The rest of the paper is structured as follows:

The system model is mentioned in Section 2. Section3 exhibits the ML estimation calculations. Joint CRB for target parameters is computed in section4. Section 5 forms a power allocation problem to minimize target tracking error by considering total power limitation. And also, a proposed SQP-based algorithm is presented in this part to solve this problem. Simulation results are shown in section 6 and finally in part 7, concluding remarks are addressed.

\section{2- System Model}

Consider a MIMO radar with widely separated antennas with $\mathrm{M}$ transmitters and $\mathrm{N}$ receivers. Denote the location of $m$ th transmitter in $\left(x_{m}, y_{m}\right)$, where $m=1,2, \ldots \ldots, M$ and the coordinates of $n$th receiver in $\left(x_{n}, y_{n}\right)$, where $n=$ $1,2, \ldots \ldots, N$. Target is in initial location $\left(x_{q}, y_{q}\right)$ with initial velocity of $\left(\dot{x}_{q}, \dot{y}_{q}\right)$. A set of low pass equivalent orthogonal waveforms, $s_{m}(t)$, is transmitted.

\footnotetext{
${ }^{1}$ sequential quadratic programming
} 
$\left(\int_{T_{m}}\left|s_{m}(t)\right|^{2} d t=1\right)$. period, effective bandwidth and transmit power of mth transmit waveform are shown as $T_{m}, \beta_{m}, P_{m}$. Target RCS corresponding to $m n$th path is modeled as a zero-mean complex Gaussian random variable $\xi_{m n} \sim C \mathcal{N}\left(0, \sigma_{m n}^{2}\right)$. Where $\sigma_{m n}^{2}$ is the variance of $m n$th path and it is known. Fig.1 shows the structure and location of antennas in MIMO radar with widely separated antennas with respect to the target.

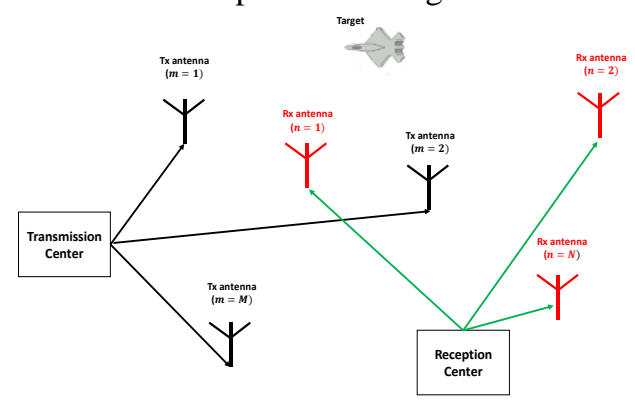

Fig. 1 configuration of a MIMO radar with widely separated antennas

We suppose the below assumption to simplify our problem.

1. $w_{m n}$ (Noise of $m n$th path with a zero-mean complex Gaussian random variable and variance of $\sigma_{w}^{2}$ ) and $\xi_{m n}$ in different paths are mutually independent.

2. Transmit waveforms are orthogonal.

$\int_{-\infty}^{+\infty} s_{m}(t) s_{m^{\prime}}^{*}(t) d t= \begin{cases}1 & \text { if } m=m^{\prime} \\ 0 & \text { if } m \neq m^{\prime}\end{cases}$

This orthogonality also remains for time delays $\tau_{m}, \tau_{m}$, and Doppler shifts $f_{d m}$ and $f_{d m}$ [26]:

$$
\begin{aligned}
& \int_{-\infty}^{+\infty} s_{m}\left(t-\tau_{m}\right) s_{m^{\prime}}^{*}\left(t-\tau_{m^{\prime}}\right) e^{j 2 \pi\left(f_{\left.d m-f_{d m^{\prime}}\right) t} d t\right.}= \\
& \left\{\begin{array}{l}
1 \quad \text { if } m=m^{\prime} \\
0 \quad \text { if } m \neq m^{\prime}
\end{array}\right.
\end{aligned}
$$

3. Set $\sigma_{w}^{2}=1$ without loss of generality.

4. The antennas are adequately separated [27]. Therefore, each path provides an independent observation of the target and $\xi_{m n}$ is independent for different $m$ and $n$ paths. The time delay of $(m, n)$ th channel in $k$ th time slot is:

$\tau_{m n, k}=\frac{d_{m, k}+d_{n, k}}{c}$

Where,

$d_{m, k} \triangleq \sqrt{\left(x_{q, k}-x_{m}\right)^{2}+\left(y_{q, k}-y_{m}\right)^{2}}$ $d_{n, k} \triangleq \sqrt{\left(x_{q, k}-x_{n}\right)^{2}+\left(y_{q, k}-y_{n}\right)^{2}}$

In the above equations, $c$ is light velocity. $d_{m, k}$ is distance from target and $m$ th transmitter and $d_{n, k}$ is distance from a target and $n$th receiver.

With these assumptions, the received signal from $m$ th transmit antenna at $n$th receive antenna at time $k$ is given by:

$r_{m n, k}(t)=\sqrt{\alpha_{m n, k} P_{m}} \xi_{m n, k}$

$s_{m}\left(t-\tau_{m n, k}\right) e^{j 2 \pi f_{m n, k} t}+w_{m n, k}(t)$

In the above equation, $w_{m n, k} \sim C \mathcal{N}\left(0, \sigma_{w}^{2}\right)$ represents a zero-mean complex Gaussian noise with the variance of $\sigma_{w}^{2}$. Power variations due to path loss is shown as $\alpha_{m n, k}=$ $\frac{1}{(4 \pi)^{3}} \frac{1}{f_{c}^{2}} \frac{1}{d_{m, k}^{2} d_{n, k}^{2}}$. Where $f_{c}$ is the carrier frequency.

Doppler frequency in $m n$ path and time $k$ is given by:

$f_{m n, k}=$
$\frac{\dot{x}_{q, k}\left(x_{m}-x_{q, k}\right)+\dot{y}_{q, k}\left(y_{m}-y_{q, k}\right)}{\lambda d_{m, k}}+\frac{\dot{x}_{q, k}\left(x_{n}-x_{q, k}\right)+\dot{y}_{q, k}\left(y_{n}-y_{q, k}\right)}{\lambda d_{n, k}}$

(6)

$\lambda$ is wavelength.

\section{2-1- Target Dynamic Model}

Target tracking in a MIMO Radar with widely separated antennas is the favorable problem of this paper. The target motion model is the constant velocity $(\mathrm{CV})$. This model is as below[7]:

$\boldsymbol{\theta}_{\mathrm{k}+1}=\mathbf{F}_{\mathrm{k}} \boldsymbol{\theta}_{\mathrm{k}}+\boldsymbol{w}_{k}^{\prime}$

Target state vector is described as $\boldsymbol{\theta}_{k}=\left[x_{q, k}, \dot{x}_{q, k}, y_{q, k}, \dot{y}_{q, k}\right]^{T}$. This parameter is considered as unknown parameter in this paper and it should be estimated. The noise $\boldsymbol{w}_{k}^{\prime}$ is a zero-mean Gaussian matrix as $\mathcal{N}\left(0, \boldsymbol{\Sigma}_{k}\right)$. Where $\mathbf{F}_{\mathrm{k}}$ is state transition matrix and $\boldsymbol{\Sigma}_{k}$ is the covariance matrix [27]:

$\mathbf{F}_{\mathrm{k}}=\left[\begin{array}{cccc}1 & T & 0 & 0 \\ 0 & 1 & 0 & 0 \\ 0 & 0 & 1 & T \\ 0 & 0 & 0 & 1\end{array}\right]$

And 


$$
\boldsymbol{\Sigma}_{k}=\left[\begin{array}{cccc}
\frac{T^{2}}{3} & \frac{T^{2}}{2} & 0 & 0 \\
\frac{T^{2}}{2} & T & 0 & 0 \\
0 & 0 & \frac{T^{2}}{3} & \frac{T^{2}}{2} \\
0 & 0 & \frac{T^{2}}{2} & T
\end{array}\right] l
$$

Where $T$ denotes sample intervals and $l$ is the density of process noise.

In next section, we compute ML estimation of $\boldsymbol{\theta}_{k}$ to use it in calculation of joint CRB.

\section{3- Maximum Likelihood Estimation}

ML estimation of the unknown parameter $\left(\boldsymbol{\theta}_{\mathrm{k}}\right)$ [28] can be achieved by testing of the likelihood ratio for two hypothesis pair, $\mathrm{H}_{1}$ is corresponding to target existence hypothesis modeled in (5) and $\mathrm{H}_{0}$ corresponding to noise only hypothesis:

$$
\begin{aligned}
& \boldsymbol{\Lambda}_{m n}\left(\boldsymbol{\theta}_{k} ; r_{m n, k}(t)\right) \\
& =\frac{1}{\sigma_{m n}^{2} P_{m}+1} \\
& \times \exp \left\{\frac{\sigma_{m n}^{2} P_{m}}{\sigma_{m n}^{2} P_{m}+1} \mid \int_{-\infty}^{+\infty} r_{m n, k}(t) s_{m}^{*}(t\right. \\
& \left.\left.-\tau_{m n, k}\right)\left.e^{-j 2 \pi f_{m n, k} t} d t\right|^{2}\right\}
\end{aligned}
$$

Where $r_{m n, k}(t)$ denotes observation signal in $n$th receiver corresponding to $m$ th transmitter.

Log-likelihood ratio based on (10) is as:

$$
\begin{aligned}
& \mathrm{L}_{m n}\left(\boldsymbol{\theta}_{k} ; r_{m n}(t)\right) \\
& =\operatorname{Ln} \Lambda_{m n}\left(\boldsymbol{\theta}_{k} ; r_{m n, k}(t)\right)=\frac{\sigma_{m n}^{2} P_{m}}{\sigma_{m n}^{2} P_{m}+1} \\
& \mid \int_{-\infty}^{+\infty} r_{m n, k}(t) s_{m}^{*}(t \\
& \left.-\tau_{m n, k}\right)\left.e^{-j 2 \pi f_{m n, k} t} d t\right|^{2}+C_{m n}
\end{aligned}
$$

Where $C_{m n}=-\ln \left(\sigma_{m n}^{2} P_{m}+1\right)$ and it does not depend to $\boldsymbol{\theta}_{k}$.

According to problem assumptions, noise and reflection coefficients (RCS) are independent, the joint time delay and Doppler likelihood ratio term are obtained by:

$$
\begin{aligned}
& \Lambda_{J}\left(\boldsymbol{\theta}_{k} ; \boldsymbol{r}(t)\right) \\
& =\prod_{m=1}^{M} \prod_{n=1}^{N} \Lambda_{m n}\left(\boldsymbol{\theta}_{k} ; r_{m n, k}(t)\right)
\end{aligned}
$$

Where $\boldsymbol{r}(t)$ collects all observed signals from all antennas set and it is introduced as:

$\boldsymbol{r}(t)=\left[r_{11}(t), r_{12}(t), \ldots, r_{N M}(t)\right]$

In the following part, joint Cramer Rao bound of target parameters is derived.

\section{4- Joint Cramer Rao Bound}

In this section, Bayesian Fisher Information and joint CRB for target position $\left(x_{q}, y_{q}\right)$ and target velocity $\left(\dot{x}_{q}, \dot{y}_{q}\right)$ under our problem assumptions are provided.

Bayesian Fisher Information provides a lower bound on the target tracking Mean Square Error (MSE) for the estimation of target state. The Cramer Rao bound represents a lower bound on the accuracy of state estimates. If $\widehat{\boldsymbol{\theta}}_{k}$ be the estimation of target state, the Cramer-Rao bound is stated as [7]:

$\mathbb{E}\left\{\left(\widehat{\boldsymbol{\theta}}_{k}-\boldsymbol{\theta}_{\mathrm{k}}\right)\left(\widehat{\boldsymbol{\theta}}_{k}-\boldsymbol{\theta}_{\mathrm{k}}\right)^{T}\right\} \geq \boldsymbol{J}_{B}^{-1}\left(\boldsymbol{\theta}_{\mathrm{k}}\right)$

Where $\mathbb{E}$ is expectation operator and $\mathbf{J}_{\mathrm{B}}\left(\boldsymbol{\theta}_{\mathrm{k}}\right)$ is the Bayesian Information Matrix (BIM).

According to [29], Bayesian Information Matrix for unknown parameter vector $\boldsymbol{\theta}_{k}$ is as:

$$
\begin{aligned}
\mathbf{J}_{\mathrm{B}}\left(\boldsymbol{\theta}_{\mathrm{k}}\right)=\left[\boldsymbol{\Sigma}_{\mathrm{k}-1}\right. & \left.+\mathbf{F} \mathbf{J}_{\mathrm{B}}^{-1}\left(\boldsymbol{\theta}_{\mathrm{k}-1}\right) \mathbf{F}^{\mathrm{T}}\right]^{-1} \\
& +\mathbb{E}\left[\mathbf{J}_{\mathrm{D}}\left(\boldsymbol{\theta}_{\mathrm{k}}\right)\right]
\end{aligned}
$$

Where $\mathbf{J}_{\mathrm{D}}$ is Fisher Information Matrix (FIM).

The first step in CRB calculation is computing FIM which is a $4 \times 4$ matrix corresponding to the second derivatives of joint log-likelihood [26]:

$$
\begin{aligned}
& \mathbf{J}_{\mathrm{D}}\left(\boldsymbol{\theta}_{\mathrm{k}}\right)= \\
& \mathbb{E}_{\boldsymbol{r}(t) ; \boldsymbol{\theta}_{k}}\left\{\nabla_{\boldsymbol{\theta}_{k}} \ln \Lambda_{J}\left(\boldsymbol{r}(t) ; \boldsymbol{\theta}_{k}\right)\left[\nabla_{\boldsymbol{\theta}_{k}} \ln \Lambda_{J}\left(\boldsymbol{r}(t) ; \boldsymbol{\theta}_{k}\right)\right]^{T}\right\} \\
& =-\mathbb{E}_{\boldsymbol{r}(t) ; \boldsymbol{\theta}_{k}}\left\{\nabla_{\boldsymbol{\theta}_{k}}\left[\nabla_{\boldsymbol{\theta}_{k}} \ln \Lambda_{J}\left(\mathbf{r}(t) ; \boldsymbol{\theta}_{k}\right)\right]^{T}\right\}
\end{aligned}
$$

Where $\mathbb{E}$ denotes the expected value operator.

By considering (10) which is the function of $\tau_{m n, k}$ and $f_{m n, k}$, a new parameter as below is described:

$$
\begin{aligned}
& \boldsymbol{\vartheta}_{k} \\
& =\left[\tau_{11, k}, \tau_{12, k}, \ldots, \tau_{N M, k}, f_{11, k}, f_{12, k}, \ldots, f_{N M, k}\right]^{T}
\end{aligned}
$$


Based on Chain rule, a new FIM is introduced as:

First, by using (17) and $\boldsymbol{\theta}_{\mathrm{k}}$, we calculate $\left(\nabla_{\boldsymbol{\theta}_{k}} \boldsymbol{\vartheta}_{k}^{T}\right)$ :

$$
\begin{aligned}
& \mathbf{J}_{\mathrm{D}}\left(\boldsymbol{\theta}_{\mathrm{k}}\right)=\left(\nabla_{\boldsymbol{\theta}_{k}} \boldsymbol{\vartheta}_{k}^{T}\right) \mathbf{J}_{\mathrm{D}}\left(\boldsymbol{\vartheta}_{k}\right)\left(\nabla_{\boldsymbol{\theta}_{k}} \boldsymbol{\vartheta}_{k}^{T}\right)^{T} \\
& \nabla_{\boldsymbol{\theta}_{k}} \boldsymbol{\vartheta}_{k}{ }^{T}=\left[\begin{array}{cccccccc}
\frac{\partial \tau_{11, k}}{\partial x_{q, k}} & \frac{\partial \tau_{12, k}}{\partial x_{q, k}} & \ldots & \frac{\partial \tau_{N M, k}}{\partial x_{q, k}} & \frac{\partial f_{11, k}}{\partial x_{q, k}} & \frac{\partial f_{12, k}}{\partial x_{q, k}} & \ldots & \frac{\partial f_{N M, k}}{\partial x_{q, k}} \\
\frac{\partial \tau_{11, k}}{\partial y_{q, k}} & \frac{\partial \tau_{12, k}}{\partial y_{q, k}} & \ldots & \frac{\partial \tau_{N M, k}}{\partial y_{q, k}} & \frac{\partial f_{11, k}}{\partial y_{q, k}} & \frac{\partial f_{12, k}}{\partial y_{q, k}} & \ldots & \frac{\partial f_{N M, k}}{\partial y_{q, k}} \\
0 & 0 & \ldots & 0 & \frac{\partial f_{11, k}}{\partial \dot{x}_{q, k}} & \frac{\partial f_{12, k}}{\partial \dot{x}_{q, k}} & \ldots & \frac{\partial f_{N M, k}}{\partial \dot{x}_{q, k}} \\
0 & 0 & \ldots & 0 & \frac{\partial f_{11, k}}{\partial \dot{y}_{q, k}} & \frac{\partial f_{12, k}}{\partial \dot{y}_{q, k}} & \ldots & \frac{\partial f_{N M, k}}{\partial \dot{y}_{q, k}}
\end{array}\right]_{4 \times 2 N M}
\end{aligned}
$$

Therefore, the above matrix parameters are defined as:

$$
\begin{aligned}
& a_{m n, k}=\frac{\partial \tau_{m n, k}}{\partial x_{q, k}}= \\
& \frac{1}{c}\left(\frac{x_{q, k}-x_{m}}{d_{m, k}}+\frac{x_{q, k}-x_{n}}{d_{n, k}}\right) \\
& b_{m n, k}=\frac{\partial \tau_{m n, k}}{\partial y_{q, k}}=\frac{1}{c}\left(\frac{y_{q, k}-y_{m}}{d_{m, k}}+\frac{y_{q, k}-y_{n}}{d_{n, k}}\right) \\
& e_{m n, k}=\frac{\partial f_{m n, k}}{\partial x_{q, k}}=-\frac{\dot{x}_{q, k}}{\lambda}\left(\frac{1}{d_{m, k}}+\frac{1}{d_{n, k}}\right)+ \\
& \frac{\left(x_{m}-x_{q, k}\right)}{\lambda\left(d_{m, k}\right)^{3}} \cdot\left[\dot{x}_{q, k}\left(x_{m}-x_{q, k}\right)+\dot{y}_{q, k}\left(y_{m}-y_{q, k}\right)\right]+ \\
& \frac{\left(x_{n}-x_{q, k}\right)}{\lambda\left(d_{n, k}\right)^{3}} \cdot\left[\dot{x}_{q, k}\left(x_{n}-x_{q, k}\right)+\dot{y}_{q, k}\left(y_{n}-y_{q, k}\right)\right] \\
& g_{m n, k}=\frac{\partial f_{m n, k}}{\partial y_{q, k}}= \\
& -\frac{\dot{y}_{q, k}}{\lambda}\left(\frac{1}{d_{m, k}}+\frac{1}{d_{n, k}}\right)+ \\
& \frac{\left(y_{m}-y_{q, k}\right)}{\lambda\left(d_{m, k}\right)^{3}} \cdot\left[\dot{x}_{q, k}\left(x_{m}-x_{q, k}\right)+\dot{y}_{q, k}\left(y_{m}-y_{q, k}\right)\right]+ \\
& \frac{\left(y_{n}-y_{q, k}\right)}{\lambda\left(d_{n, k}\right)^{3}} \cdot\left[\dot{x}_{q, k}\left(x_{n}-x_{q, k}\right)+\dot{y}_{q, k}\left(y_{n}-y_{q, k}\right)\right] \\
& V_{m n, k}=\frac{\partial f_{m n, k}}{\partial \dot{x}_{q, k}}=\frac{x_{m}-x_{q, k}}{\lambda d_{m, k}}+\frac{x_{n}-x_{q, k}}{\lambda d_{n, k}} \\
& q_{m n, k}=\frac{\partial f_{m n, k}}{\partial \dot{y}_{q, k}}= \\
& \frac{y_{m}-y_{q, k}}{\lambda d_{m, k}}+\frac{y_{n}-y_{q, k}}{\lambda d_{n, k}}
\end{aligned}
$$

As shown in the above equations, the parameters of $a_{m n, k}$ ، $b_{m n, k} \cdot e_{m n, k} \cdot g_{m n, k} \cdot V_{m n, k} \cdot q_{m n, k}$ are determined by target position and velocity and $c$ is light velocity.

If $\nabla_{\boldsymbol{\theta}_{k}} \boldsymbol{\vartheta}_{k}{ }^{T}$ is divided into matrix blocks as:

$$
\nabla_{\boldsymbol{\theta}_{k}} \boldsymbol{\vartheta}_{k}{ }^{T}=\left[\begin{array}{ll}
\mathbf{A} & \mathbf{B} \\
\mathbf{0} & \mathbf{D}
\end{array}\right]
$$

$\mathbf{A}, \mathbf{B}, \mathbf{D}$ are $2 \times N M$ matrices and $\mathbf{0}$ is $2 \times N M$ zero matrix. $\mathbf{J}_{\mathrm{D}}\left(\boldsymbol{\vartheta}_{k}\right)$ is also obtained as:

$\mathbf{J}_{\mathrm{D}}\left(\boldsymbol{\vartheta}_{k}\right)=-\mathbb{E}_{\boldsymbol{r}(t) ; \boldsymbol{\theta}_{k}}\left\{\nabla_{\boldsymbol{\vartheta}_{k}}\left[\nabla_{\boldsymbol{\vartheta}_{k}} \ln \Lambda_{J}\left(\mathbf{r}(t) ; \boldsymbol{\theta}_{k}\right)\right]^{T}\right\}$

Where it is a $2 N M \times 2 N M$ matrix.

$\mathbf{J}_{\mathrm{D}}\left(\boldsymbol{\vartheta}_{k}\right)$ is represented as:

$\mathbf{J}_{\mathrm{D}}\left(\boldsymbol{\vartheta}_{k}\right)=\left[\begin{array}{ll}\mathbf{J}_{\mathrm{D}}^{U L} & \mathbf{J}_{\mathrm{D}}^{U R} \\ \mathbf{J}_{\mathrm{D}}^{L L} & \mathbf{J}_{\mathrm{D}}^{L R}\end{array}\right]$

Where $\mathbf{J}_{\mathrm{D}}^{U L}, \mathbf{J}_{\mathrm{D}}^{U R}, \mathbf{J}_{\mathrm{D}}^{L L}$, and $\mathbf{J}_{\mathrm{D}}^{L R}$ are $N M \times N M$ matrices. In fact, $\mathbf{J}_{\mathrm{D}}^{U L}$ includes all second-order derivatives terms with respect to $\tau_{m n, k}$ for all $m$ and $n$ in time slot $k$. $\mathbf{J}_{\mathrm{D}}^{U R}, \mathbf{J}_{\mathrm{D}}^{L L}$ contains second-order derivatives with respect to $\tau_{m n, k}$ and $f_{m n, k}$ for all $m$ and $n$ in time slot $k$. $\mathbf{J}_{\mathrm{D}}^{L R}$ contains second-order derivatives with respect to $f_{m n, k}$ for all $m$ and $n$ in time slot $k$.

Therefore,

$\mathbf{J}_{\mathrm{D}}^{U L}=\boldsymbol{C} \odot\left(\boldsymbol{I}_{N} \otimes \operatorname{diag}\left\{\epsilon_{1}, \epsilon_{2}, \ldots, \epsilon_{M}\right\}\right)$

Where $\boldsymbol{I}_{N}$ is an $N \times N$ identity matrix, $\odot$ is Hadmard product operator, and $\Theta$ is Kronecker product operator.

$\boldsymbol{C}=8 \pi^{2} \operatorname{diag}\left\{\frac{\sigma_{11}^{4} P_{1}^{2}}{\sigma_{11}^{2} P_{1}+1}, \ldots ., \frac{\sigma_{M N}^{4} P_{M}^{2}}{\sigma_{M N}^{2} P_{M}+1}\right\}$

In fact, $\boldsymbol{C}$ is an $N M \times N M$ matrix.

$\mathbf{J}_{\mathrm{D}}^{U R}=\mathbf{J}_{\mathrm{D}}^{L L}=$

C○diag $\left\{\gamma_{11, k}, \gamma_{12, k}, \ldots, \gamma_{N M, k}\right\}$

$\mathbf{J}_{\mathrm{D}}^{L R}=\boldsymbol{C} \odot \operatorname{diag}\left\{\eta_{11, k}, \eta_{12, k}, \ldots, \eta_{N M, k}\right\}$

Where $\epsilon_{M}, \gamma_{m n, k}$ and $\eta_{m n, k}$ depend on receive waveform characteristics and they are defined as: 


$$
\begin{aligned}
\epsilon_{m}=\int_{-\infty}^{+\infty} f^{2}\left|S_{m}(f)\right|^{2} d f \\
-\left.\left.\left|\int_{-\infty}^{+\infty} f\right| S_{m}(f)\right|^{2} d f\right|^{2}
\end{aligned}
$$

$\gamma_{m n, k}=\frac{1}{2 \pi}$

$\mathcal{F}\left\{\int_{-\infty}^{+\infty} t s_{m}^{*}\left(t-\tau_{m n, k}\right) \frac{\partial s_{m}\left(t-\tau_{m n, k}\right)}{\partial \tau_{m n, k}} d t\right\}-$

$\int_{-\infty}^{+\infty} f\left|S_{m}(f)\right|^{2} d f \cdot \int_{-\infty}^{+\infty} t\left|s_{m}\left(t-\tau_{m n, k}\right)\right|^{2} d t$

$$
\begin{aligned}
& \int_{-\infty}^{\eta_{m n, k}}= \\
& \int^{+\infty} t^{2}\left|s_{m}\left(t-\tau_{m n, k}\right)\right|^{2} d t \\
& \quad-\left.\left.\left|\int_{-\infty}^{+\infty} t\right| s_{m}\left(t-\tau_{m n, k}\right)\right|^{2} d t\right|^{2}
\end{aligned}
$$

Therefore, By considering the right-hand matrix as $\mathrm{H}_{4 \times 4}$ which is the function of target position and velocity in each $m n$ path and in different frames (although this matrix is not the function of transmit power), (36) can be reform as:

$$
\mathbf{J}_{\mathrm{D}}\left(\boldsymbol{\theta}_{\mathrm{k}}\right)=\sum_{m=1}^{M} \sum_{n=1}^{N} 8 \pi^{2} \frac{\sigma_{m n}^{4} P_{m}^{2}}{\sigma_{m n}^{2} P_{m}+1} \cdot\left(\mathrm{H}_{\mathrm{mn}}\right)_{4 \times 4}
$$

In fact, $\mathbf{J}_{\mathrm{D}}\left(\boldsymbol{\theta}_{\mathrm{k}}\right)$ is a $4 \times 4$ matrix that is the function of transmit power.

Note that CRB for unknown parameter estimation is achieved by the diagonal elements of the inverse of FIM :

$$
\begin{aligned}
& \operatorname{var}\left(\hat{x}_{q, k}\right) \geq\left[\mathbf{J}_{\mathrm{D}}^{-1}\left(\boldsymbol{\theta}_{\mathrm{k}}\right)\right]_{1,1} \\
& \operatorname{var}\left(\hat{y}_{q, k}\right) \geq\left[\mathbf{J}_{\mathrm{D}}^{-1}\left(\boldsymbol{\theta}_{\mathrm{k}}\right)\right]_{2,2} \\
& \operatorname{var}\left(\hat{x}_{q, k}\right) \geq\left[\mathbf{J}_{\mathrm{D}}^{-1}\left(\boldsymbol{\theta}_{\mathrm{k}}\right)\right]_{3,3} \\
& \operatorname{var}\left(\hat{\dot{y}}_{q, k}\right) \geq\left[\mathbf{J}_{\mathrm{D}}^{-1}\left(\boldsymbol{\theta}_{\mathrm{k}}\right)\right]_{4,4}
\end{aligned}
$$

In above equations $\operatorname{var}\left(\hat{x}_{q, k}\right)$,var $\left(\hat{y}_{q, k}\right)$, var $\left(\hat{\dot{x}}_{q, k}\right)$ and $\operatorname{var}\left(\hat{\dot{y}}_{q, k}\right)$ respectively show the variance of target position in axis of $x$ and $y$ and also target velocity in axis of $x$ and $y$ in $k$ th frame.In next section, our power allocation problem is formed.

In the above equations, $S_{m}(f)$ is a Fourier transform of $s_{m}(t)$. Therefore, (36) is obtained.

\section{5- Power Allocation Problem}

In this section, we present power allocation strategy for a MIMO radar with widely separated antennas. In this problem and in the formulation of objective function, we consider a random RCS for target. This is rarely done in other papers and for simplicity, they consider deterministic reflection coefficients for target in MIMO radar with widely separated antennas. So this assumption make our main part of our objective function (equation (37)) complex.

\section{5-1- Problem Formulation}

In definition of our power allocation problem, we consider trace of CRB matrix as target tracking error and it is shown as:

$\mathbb{F}\left(\boldsymbol{\theta}_{\mathrm{k}}, \mathbf{P}\right)=\operatorname{trace}\left(\mathbf{Y}_{k} \mathbf{J}_{\mathrm{D}}^{-1}\left(\boldsymbol{\theta}_{\mathrm{k}}\right)\right)$

Where $\mathbf{P}=\left[P_{1}, \ldots, P_{M}\right]$ is a transmit power vector, $\mathbf{Y}_{k}$ is the normalization matrix and $\mathbb{F}$ is considered as the target tracking error.

Note that in equation (15), the first term (left-hand term) is approximately constant, therefore the second part, $\mathbf{J}_{\mathrm{D}}\left(\boldsymbol{\theta}_{\mathrm{k}}\right)$, is utilized for the calculation of the Another notable point is about diagonal elements of $\mathbf{J}_{\mathrm{D}}^{-1}\left(\boldsymbol{\theta}_{\mathrm{k}}\right)$, since the first and third diagonal elements are corresponding to the target position and second and fourth diagonal elements are corresponding to target velocity and their scales are different, so it is important to change them with no loss of generality to have the same scale. Fisher information matrix.Therefore we introduce $\boldsymbol{Y}_{k}$ as:

$\mathbf{Y}_{k}=I_{2} \otimes\left[\begin{array}{ll}1 & 0 \\ 0 & T\end{array}\right]$

Where $I_{2}$ is $2 \times 2$ identity matrix and $\otimes$ is the Kronecker product operator.

$$
\begin{gathered}
\mathbf{J}_{\mathbf{D}}\left(\boldsymbol{\theta}_{\mathbf{k}}\right)=\sum_{m=1}^{M} \sum_{n=1}^{N} 8 \pi^{2} \frac{\sigma_{m n}^{4} P_{m}^{2}}{\sigma_{m n}^{2} P_{m}+1} \times \\
{\left[\begin{array}{cccc}
\epsilon_{m} a_{m n, k}^{2}+2 \gamma_{m n, k} a_{m n, k} e_{m n, k}+\eta_{m n, k} e_{m n, k}^{2} & \left(\epsilon_{m} a_{m n, k}+\gamma_{m n, k} e_{m n, k}\right) b_{m n, k}+\left(\gamma_{m n, k} a_{m n, k}+\eta_{m n, k} e_{m n, k}\right) g_{m n, k} & \left(\gamma_{m n, k} a_{m n, k}+\eta_{m n, k} e_{m n, k}\right) V_{m n, k} & \left(\gamma_{m n, k} a_{m n, k}+\eta_{m n, k} e_{m n, k}\right) q_{m n, k} \\
\left(\epsilon_{m} a_{m n, k}+\gamma_{m n, k} e_{m n, k}\right) b_{m n, k}+\left(\gamma_{m n, k} a_{m n, k}+\eta_{m n, k} e_{m n, k}\right) g_{m n, k} & \epsilon_{m} b_{m n, k}^{2}+2 \gamma_{m n, k} b_{m n, k} g_{m n, k}+\eta_{m n, k} g_{m n, k}^{2} & \left(\gamma_{m n, k} b_{m n, k}+\eta_{m n, k} g_{m n, k}\right) V_{m n, k} & \left(\gamma_{m n, k} b_{m n, k}+\eta_{m n, k} g_{m n, k}\right) q_{m n, k} \\
\left(\gamma_{m n, k} a_{m n, k}+\eta_{m n, k} e_{m n, k}\right) V_{m n, k} & \left(\gamma_{m n, k} b_{m n, k}+\eta_{m n, k} g_{m n, k}\right) V_{m n, k} & \eta_{m n, k} V_{m n, k}^{2} & \eta_{m n, k} V_{m n, k} q_{m n, k} \\
\left(\gamma_{m n, k} a_{m n, k}+\eta_{m n, k} e_{m n, k}\right) q_{m n, k} & \left(\gamma_{m n, k} b_{m n, k}+\eta_{m n, k} g_{m n, k}\right) q_{m n, k} & \eta_{m n, k} V_{m n, k}
\end{array}\right]}
\end{gathered}
$$


Therefore, the power allocation problem for target tracking in MIMO Radar with widely separated antennas by considering random radar cross-section (RCS) is defined as:

$$
\begin{aligned}
& \min _{P_{m}} \mathbb{F}\left(\boldsymbol{\theta}_{\mathrm{k}}, \mathbf{P}\right) \\
& \text { s.t. } \quad \sum_{m=1}^{M} P_{m} \leq P_{T} \\
& \quad P_{\text {min }} \leq P_{m} \leq P_{\text {max }} \quad, m=1,2, \ldots, M
\end{aligned}
$$

In the above problem, the first constraint represents that total transmit power is less than a predetermined value, $P_{T}$. In fact, it says that this value does not exceed $P_{T}$, because MIMO radar tries to use the least power for target tracking and it is not intercepted by other radars. The second constraint shows that each antenna also has power limitation.

\section{5-2- Problem Solving}

The problem (44) is nonlinear and it is nonconvex, So we propose the sequential quadratic programming (SQP) algorithm to solve it.

\section{5-2-1 SQP Method}

Sequential quadratic programming methods are famous to be affective for solving a series of related nonlinear optimization problems because of favorable hot and warm start properties - a solution for one problem is a good estimate of the solution of the next.

SQP applies Newton's method (or quasi-Newton methods) to directly solve the KKT conditions for the original problem. As a result, the accompanying subproblem turns out to be the minimization of a quadratic approximation to the Lagrangian function subject to a linear approximation to the constraints. Therefore, this kind of process is also known as a projected Lagrangian, or the NewtonLagrange, approach. By its nature, this method produces both primal and dual (Lagrange multiplier) solutions. The procedure of SQP for constrained nonlinear problem as below [28]:

Minimize: $f(\boldsymbol{x})$

Subject to: $g_{i}(\boldsymbol{x}) \leq 0, \quad i=1, \ldots, n_{g}$

$$
h_{i}(\boldsymbol{x})=0, \quad i=1, \ldots, n_{h}
$$$$
x^{l}<x<x^{u}
$$

Where, $f$ is an objective function, $g$ and $h$ represent inequality and equality function and $f, g$, and $h$ are twice continuously differentiable. $\boldsymbol{x}$ shows the desirable variable matrix and it is limited by lower and upper bound $x^{l}$ and $x^{u}$.

Given an iterate $\left(\boldsymbol{x}^{k}, \boldsymbol{\lambda}^{k}, \boldsymbol{v}^{k}\right)$, where $\lambda^{k}$ and $\boldsymbol{v}^{k} \geq 0$ are the Lagrange multiplier estimates for the equality and inequality constraints, respectively, consider the following QP subproblem as a direct extension of $\mathrm{QP}\left(\boldsymbol{x}^{k}, \boldsymbol{\lambda}^{k}\right)$ :

$$
\begin{aligned}
\min _{\boldsymbol{d}^{k}} f\left(\boldsymbol{x}^{k}\right)+\boldsymbol{\nabla} f\left(\boldsymbol{x}^{k}\right)^{T} \boldsymbol{d}^{k}+\frac{1}{2} \boldsymbol{d}^{k^{T}} \boldsymbol{\nabla}_{x x}^{2} \mathcal{L}\left(\boldsymbol{x}^{k}, \lambda^{k}, \boldsymbol{v}^{k}\right) \boldsymbol{d}^{k} \\
\text { s.t. } g_{i}(\boldsymbol{x})+\nabla g_{i}\left(\boldsymbol{x}^{k}\right)^{T} \boldsymbol{d}^{k}=0, \quad i=1, \ldots, n_{g} \\
h_{i}(\boldsymbol{x})+\boldsymbol{\nabla} h_{i}\left(\boldsymbol{x}^{k}\right)^{T} \boldsymbol{d}^{k}=0, \quad i=1, \ldots, n_{h}
\end{aligned}
$$

(51) is named as $\mathrm{QP}\left(\boldsymbol{x}^{k}, \boldsymbol{\lambda}^{k}, \boldsymbol{v}^{k}\right)$ and $\mathcal{L}\left(\boldsymbol{x}^{k}, \boldsymbol{\lambda}^{k}, \boldsymbol{v}^{k}\right)=$ $f(\boldsymbol{x})+\boldsymbol{v}^{T} \boldsymbol{g}(\boldsymbol{x})+\lambda^{T} \boldsymbol{h}(\boldsymbol{x})$.

Note that in above equations, for simplicity the constraint is not considered and also note that the KKT

Conditions for $\mathrm{QP}\left(\boldsymbol{x}^{k}, \boldsymbol{\lambda}^{k}, \boldsymbol{v}^{k}\right)$ need that, in addition to primal feasibility, Lagrange multipliers $\lambda^{k+1}, \boldsymbol{v}^{k+1}$ be found like that:

$$
\begin{gathered}
\boldsymbol{\nabla} f\left(\boldsymbol{x}^{k}\right)+\nabla_{x x}^{2} \mathcal{L}\left(\boldsymbol{x}^{k}, \lambda^{k}, \boldsymbol{v}^{k}\right) \boldsymbol{d}^{k}+ \\
\boldsymbol{\nabla} \boldsymbol{g}\left(\boldsymbol{x}^{k}\right)^{T} \boldsymbol{v}^{k+1}+\boldsymbol{\nabla h}\left(\boldsymbol{x}^{k}\right)^{T} \lambda^{k+1}=0 \\
{\left[\boldsymbol{g}\left(\boldsymbol{x}^{k}\right)+\boldsymbol{\nabla} \boldsymbol{g}\left(\boldsymbol{x}^{k}\right)^{T} \boldsymbol{d}^{k}\right]^{T} \boldsymbol{v}^{k+1}=0}
\end{gathered}
$$

With $\boldsymbol{v}^{k+1} \geq 0$ and $\lambda^{k+1}$ unrestricted in sign. Clearly, if $\boldsymbol{d}^{k}=0$, then $\boldsymbol{x}^{k}$ and $\boldsymbol{\lambda}^{k+1}$ and $\boldsymbol{v}^{k+1}$ yields KKT conditions to original problem. Otherwise, we should set $\boldsymbol{x}^{k+1}=\boldsymbol{x}^{k}+\boldsymbol{d}^{k}$ as before, increment $k$ by 1 and repeat the procedure.

\section{5-2-2 Proposed SQP-based Power Allocation Algorithm}

As mentioned before, Problem (44) subject to (45-46) is nonconvex and it should be solved by nonconvex suitable algorithms. In this section, we proposed SQP-based power allocation algorithm for our desired problem. In our scenario, problem (44) is our objective function and $P_{m}$ is our desired variable. In fact, (44) is $f$ in (47) and $\mathbf{P}$ in our problem is $\boldsymbol{x}$ in (47). By considering $\sum_{m=1}^{M} P_{m}-P_{T} \leq 0$, we can claim that $\boldsymbol{g}(\boldsymbol{x})$ in (48) is equal to $\sum_{m=1}^{M} P_{m}-P_{T}$ in our problem.

There is not constraint (49) is our problem and $x^{l}=\boldsymbol{P}_{\min }$ and $x^{u}=\boldsymbol{P}_{\text {max }}$.

Therefore, our proposed algorithm is based on previous section and it is proposed as:

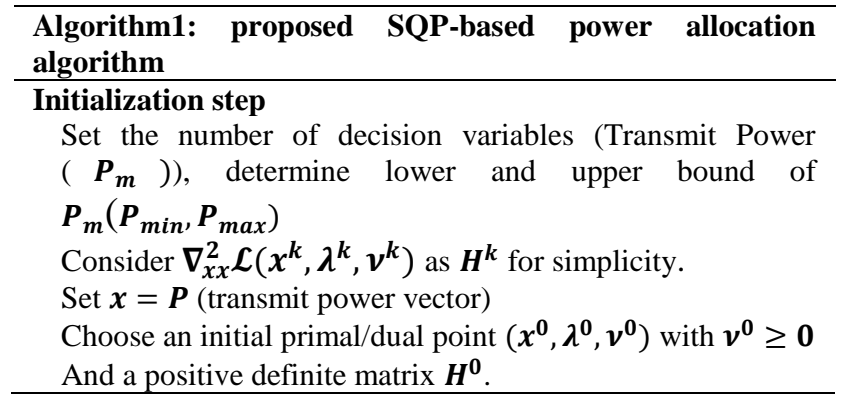


Case2

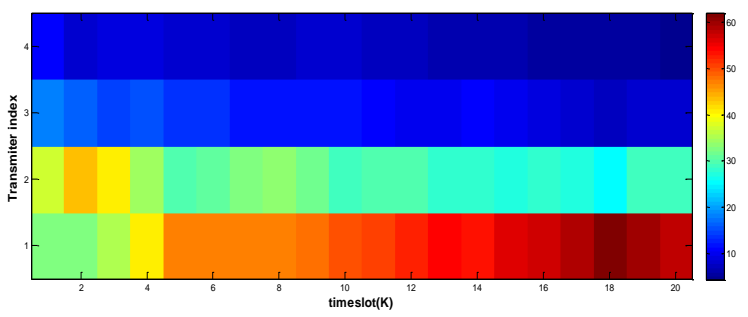

Fig. 4 Transmit power of each transmit antenna (\%) for two cases with respect to timeslot

By attention to Fig.4, we can realize that by closing the target to the transmitter, more power is assigned to the transmitter. Therefore, in Case1, the transmitter 1 and 4, in Case2, the transmitter 1and 2, take more power to perform better target tracking performance.

Fig.5 exhibits that the proposed SQP-based power allocation algorithm has better performance than other strategies (Random and uniform power allocation) and PSO-based algorithm. it shows that the CRB of target tracking error is the least when the proposed SQP-based algorithm is used to perform the power allocation strategy. This priority is seen in all cases (Case1 (Fig.5 (a)), Case2 (Fig.5 (b))). Therefore, we can conclude that the proposed strategy has the best performance.
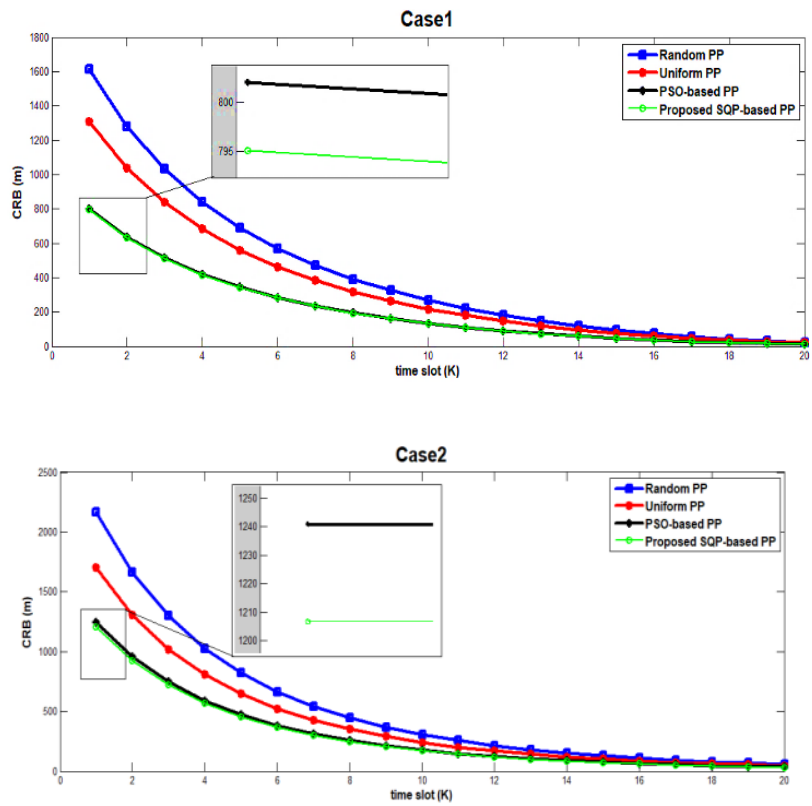

Fig.5. the comparison of the proposed SQP-based power allocation algorithm with uniform and random power allocation strategies in all two cases.

Table 2 illustrates the time duration which SQP-based and PSO-based algorithm need to run the simulations of our scenarios. Note that all the simulations are performed in system with intel(R) core(TM)i7-3612QM CPU @ $2.1 \mathrm{GHz}$ and 6 GB RAM.
Table 2: time cost of the proposed SQP-based and PSO-based algorithm

\begin{tabular}{|c|c|c|c|}
\hline Cases & $\begin{array}{c}\text { SQP-based } \\
\text { algorithm } \\
\text { (s) }\end{array}$ & $\begin{array}{c}\text { PSO-based } \\
\text { algorithm } \\
\text { (s) }\end{array}$ & $\begin{array}{c}\text { Maximum } \\
\text { iteration }\end{array}$ \\
\hline Case1 & 45.904317 & 505.183312 & 1000 \\
\hline Case 2 & 45.512462 & 504.914239 & 1000 \\
\hline
\end{tabular}

The above table shows that the proposed algorithm has more efficiency in real time scenarios. It is very crucial in target tracking scenarios because target is moving quickly in environment and the radar should track it simultaneously.

To show the impact of the number of antennas on MIMO radar target tracking performance, we consider another scenario, Case3, with $M=6, N=6$ for geometry deployment like Case1. Fig.6 demonstrates that increasing the antennas improves the performance of target tracking.

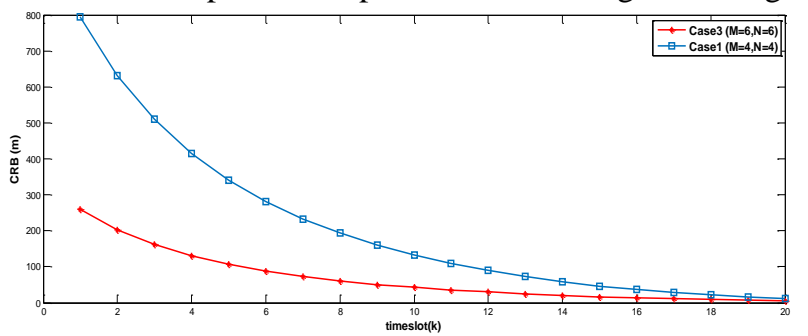

Fig.6. impact of the number of antennas on MIMO radar target tracking performance (with SQP-based algorithm)

To prove the use of the proposed tracking procedures, Fig.7 shows the target tracking RMSE and CRB in for Cases. The RMSE is computed as:

$\mathrm{RMSE}_{\mathrm{k}}$

$=\sqrt{\frac{1}{N_{M C}} \sum_{i=1}^{N_{M C}} \operatorname{trace}\left(\mathbf{Y}_{k}\left(\boldsymbol{\theta}_{k}-\widehat{\boldsymbol{\theta}}_{k}^{j}\right)\left(\boldsymbol{\theta}_{k}-\widehat{\boldsymbol{\theta}}_{k}^{j}\right)^{T} \mathbf{Y}_{k}^{T}\right)}$

Where $N_{M C}$ is the number of Monte Carlo trials and $\widehat{\boldsymbol{\theta}}_{k}^{j}$ denotes the state estimate of the target in the $j$ th trial.

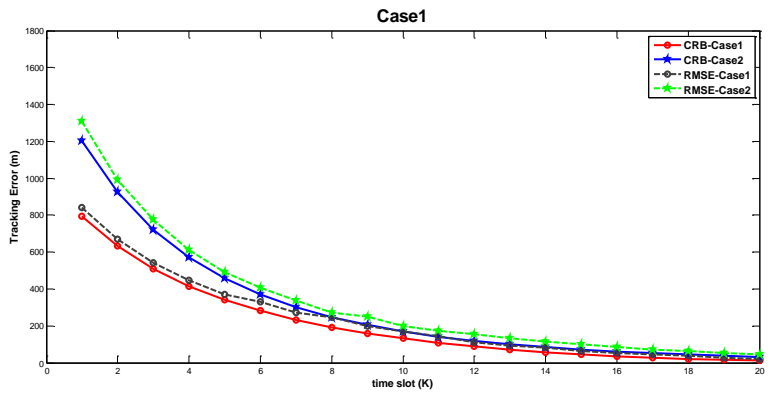

Fig.7. the evaluation of target tracking errors in two cases with proposed SQP-based target tracking algorithm 
The proximity of CRB and RMSE results in Fig.7, exhibits that the proposed target tracking procedure is very near to actual conditions.

We consider random RCS model for target. Therefore we compare deterministic RCS and random RCS in performance of power allocation in target tracking in MIMO radar in Fig8. Fig 8 shows that by considering random RCS for power allocation strategy in MIMO radar, the target tracking error is decreased. This experiment is performed for Case1.

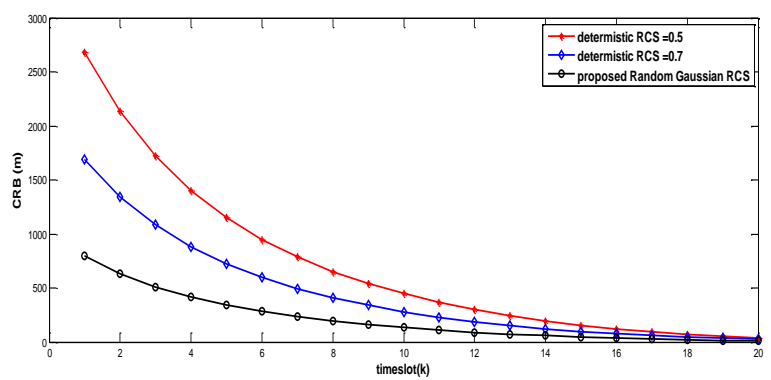

Fig.8. impact of deterministic and proposed random Gaussian RCS model on target tracking error

\section{7- Conclusions}

In this paper, a MIMO radar with widely separated antennas is considered to investigate power allocation strategy performance. A zero-mean random complex Gaussian with random variance in different transmit-

\section{References}

[1] M. A. Darzikolaei, A. Ebrahimzade, and E. Gholami, "Classification of radar clutters with artificial neural network," in 2015 2nd International Conference on Knowledge-Based Engineering and Innovation (KBEI), 2015, pp. 577-581.

[2] M. A. Darzikolaei, A. Ebrahimzade, and E. Gholami, "The Separation of Radar Clutters using Multi-Layer Perceptron," Information Systems \& Telecommunication, p. 41, 2017.

[3] E. Fishler, A. Haimovich, R. Blum, D. Chizhik, L. Cimini, and R. Valenzuela, "MIMO radar: An idea whose time has come," in Proceedings of the 2004 IEEE Radar Conference (IEEE Cat. No. 04CH37509), 2004, pp. 71-78.

[4] E. Fishler, A. Haimovich, R. S. Blum, L. J. Cimini, D. Chizhik, and R. A. Valenzuela, "Spatial diversity in radarsModels and detection performance," IEEE Transactions on signal processing, vol. 54, no. 3, pp. 823-838, 2006.

[5] J. Li and P. Stoica, "MIMO radar with colocated antennas," IEEE Signal Processing Magazine, vol. 24, no. 5, pp. 106114,2007

[6] A. M. Haimovich, R. S. Blum, and L. J. Cimini, "MIMO radar with widely separated antennas," IEEE Signal Processing Magazine, vol. 25, no. 1, pp. 116-129, 2007.

[7] M. Hai, "MIMO radar with widely separated antennas technology," IEE Signal Magazine, vol. 26, no. 2, pp. 98106, 2009. receive paths is considered for target RCS. The simulation results demonstrate that this assumption enhances the MIMO radar with widely separated antennas performance. Using joint estimation of target position and velocity tracking error in power allocation problems for target tracking in MIMO radar with widely separated antennas is the another novel idea which is used in this paper.

In simulations, two different antenna geometry deployments with the different number of antennas (Case1 and 2) is considered to assess the proposed structure for power allocation problem. This paper demonstrates the power allocation problem to minimize the target tracking errors subject to the limitation in total transmit power and power of each transmit antenna using MIMO radar with widely separated antennas. Due to the nonconvexity of this problem, the SQP-based power allocation algorithm is proposed. The simulation experiments are performed in various conditions and simulation results exhibits the accuracy of the proposed algorithm.

Multi target tracking problem will be our future challenge to improve our work. In additions, we will add target RCS estimation to target state estimation vector to enhance target tracking performance in MIMO radar with widely separated antennas.

[8] Y. Bar-Shalom, X. R. Li, and T. Kirubarajan, Estimation with applications to tracking and navigation: theory algorithms and software. John Wiley \& Sons, 2004.

[9] A. Pakdaman and H. Bakhshi, "Separable transmit beampattern design for MIMO radars with planar colocated antennas," AEU-International Journal of Electronics and Communications, vol. 89, pp. 153-159, 2018.

[10] M. Xie, W. Yi, T. Kirubarajan, and L. Kong, "Joint node selection and power allocation strategy for multitarget tracking in decentralized radar networks," IEEE Transactions on Signal Processing, vol. 66, no. 3, pp. 729-743, 2017.

[11] H. Godrich, A. P. Petropulu, and H. V. Poor, "Power allocation strategies for target localization in distributed multiple-radar architectures," IEEE Transactions on Signal Processing, vol. 59, no. 7, pp. 3226-3240, 2011.

[12] H. Chen, S. Ta, and B. Sun, "Cooperative game approach to power allocation for target tracking in distributed MIMO radar sensor networks," IEEE Sensors Journal, vol. 15, no. 10, pp. 5423-5432, 2015.

[13] P. Chavali and A. Nehorai, "Scheduling and power allocation in a cognitive radar network for multiple-target tracking," IEEE Transactions on Signal Processing, vol. 60, no. 2, pp. 715-729, 2011.

[14] C. Shi, S. Salous, F. Wang, and J. Zhou, "Power allocation for target detection in radar networks based on low probability of intercept: A cooperative game theoretical strategy," Radio Science, vol. 52, no. 8, pp. 1030-1045, 2017. 
[15] J. Yan, H. Liu, B. Jiu, and Z. Bao, "Power allocation algorithm for target tracking in unmodulated continuous wave radar network," IEEE sensors journal, vol. 15, no. 2, pp. 1098-1108, 2014.

[16] L. Wang, L. Wang, Y. Zeng, and M. Wang, "Jamming power allocation strategy for MIMO radar based on MMSE and mutual information," IET Radar, Sonar \& Navigation, vol. 11, no. 7, pp. 1081-1089, 2017.

[17] S. M. H. Andargoli and J. Malekzadeh, "LPI radar network optimization based on geometrical measurement fusion," Optimization and Engineering, vol. 20, no. 1, pp. 119-150, 2019.

[18] B. Ma, H. Chen, B. Sun, and H. Xiao, "A joint scheme of antenna selection and power allocation for localization in MIMO radar sensor networks," IEEE communications letters, vol. 18, no. 12, pp. 2225-2228, 2014.

[19] X. Li, W. Yi, G. Cui, L. Kong, and X. Yang, "Radar selection for single-target tracking in radar networks," in 2015 IEEE Radar Conference (RadarCon), 2015, pp. 05450550 .

[20] Y. Lu, Z. He, X. Zhang, and S. Liu, "Transmit and receive sensors joint selection for MIMO radar tracking based on PCRLB," in 2016 IEEE 13th International Conference on Signal Processing (ICSP), 2016, pp. 1551-1555.

[21] J. She, F. Wang, and J. Zhou, "A novel sensor selection and power allocation algorithm for multiple-target tracking in an LPI radar network," Sensors, vol. 16, no. 12, p. 2193, 2016.

[22] J. Yan, H. Liu, W. Pu, S. Zhou, Z. Liu, and Z. Bao, "Joint beam selection and power allocation for multiple target tracking in netted colocated MIMO radar system," IEEE Transactions on Signal Processing, vol. 64, no. 24, pp. 64176427, 2016.

[23] X. Song, N. Zheng, and T. Bai, "Resource allocation schemes for multiple targets tracking in distributed MIMO radar systems," International Journal of Antennas and Propagation, vol. 2017, 2017.

[24] N. Garcia, A. M. Haimovich, M. Coulon, and M. Lops, "Resource allocation in MIMO radar with multiple targets for non-coherent localization," IEEE Transactions on Signal Processing, vol. 62, no. 10, pp. 2656-2666, 2014.

[25] Yi, Wei, Ye Yuan, Reza Hoseinnezhad, and Lingjiang Kong. "Resource scheduling for distributed multi-target tracking in netted colocated MIMO radar systems." IEEE Transactions on Signal Processing 68 (2020): 1602-1617.

[26] Li, Zhengjie, Junwei Xie, Haowei Zhang, Houhong Xiang, and Zhaojian Zhang. "Adaptive sensor scheduling and resource allocation in netted collocated MIMO radar system for multi-target tracking." IEEE Access 8 (2020): 109976109988.

[27] Q. He, R. S. Blum, and A. M. Haimovich, "Noncoherent MIMO radar for location and velocity estimation: More antennas means better performance," IEEE Transactions on Signal Processing, vol. 58, no. 7, pp. 3661-3680, 2010.

[28] V. Trees and L. Harry, Detection, Estimation, and Modulation Theory-Part 1-Detection, Estimation, and Linear Modulation Theory. John Wiley \& Sons New York, 2001.

[29] H. Godrich, A. M. Haimovich, and R. S. Blum, "Target localization accuracy gain in MIMO radar-based systems," IEEE Transactions on Information Theory, vol. 56, no. 6, pp. 2783-2803, 2010
[30] D. Wassel, "Exploring novel designs of nlp solvers: architecture and implementation of worhp," $\mathrm{PhD}$ Thesis, Universität Bremen, 2013.

Mohammad Akhondi Darzikolaei received a B.Sc and M.Sc degree from the Babol Noshirvani University of Technology. $\mathrm{He}$ worked on radar clutters and radar signal processing. He is now a Telecommunication Ph.D. candidate at the Babol Noshirvani University of Technology. His research focuses on radar and sonar signal processing, speech processing and pattern recognition, and artificial neural networks.

Mohammad Reza Karami-Mollaei received the B.Sc in Electrical and Electronic Engineering in 1992, M.Sc of signal processing in 1994, and Ph.D in 1998 in Biomedical Engineering from I.N.P.L d'Nancy of France. He is now an associate professor with the Department of Electrical and Computer Engineering, Babol University of Technology. Since 1998 his research is in signal and speech processing. He teaches Digital Signal, Biomedical and speech processing at university. His research interests include Speech, Image and signal processing.

Maryam Najimi received her B.Sc in electronics from Sistanand Baloochestan University,Zahedan,Iran in 2004 and her M.Sc. in telecommunication systems engineering from K.N.Toosi University of Technology, Tehran, Iran, and Ph.D. degree in communication from Babol University of Technology, Mazandaran, Iran, in 2008 and 2014, respectively. She is currently an assistant professor with the department of electrical and computer engineering,University of Science and Technology, Behshahr, Iran. Her interests include Spectrum sensing in wireless cognitive sensor networks. 